\title{
Prévalence et variation de la consommation de tabac à rouler chez les jeunes Canadiens depuis 2008
}

\author{
A. G. Cole, M. Sc. (1); S. T. Leatherdale, Ph. D. (1); V. L. Rynard, M. Sc. (2)
}

Cet article a fait l'objet d'une évaluation par les pairs.

Diffuser cet article sur Twitter

\section{Résumé}

Introduction : Les cigarettes roulées représentent une solution de rechange abordable par rapport aux cigarettes industrielles, en particulier pour les jeunes à faible revenu. Cette étude a pour but de caractériser les variations de prévalence de la consommation de tabac à rouler des jeunes Canadiens entre 2008 et 2010 et d'examiner les caractéristiques sociodémographiques associées à cette consommation en 2010.

Méthodologie : Cette étude utilise des données représentatives recueillies chez des élèves de la $9^{\mathrm{e}}$ à la $12^{\mathrm{e}}$ année dans le cadre des cycles 2008-2009 et 2010-2011 de l'Enquête sur le tabagisme chez les jeunes (ETJ).

Résultats : Parmi les fumeurs, 30,5 \% consommaient des cigarettes roulées. Les jeunes bénéficiant d'un revenu disponible de plus de 100 \$ par semaine étaient moins nombreux à consommer ce type de produit (rapport de cotes $[\mathrm{RC}]=0,49$; intervalle de confiance [IC] à $95 \%: 0,34$ à 0,71 ). Les consommateurs de tabac à rouler étaient plus nombreux à consommer également de l'alcool ( $\mathrm{RC}=2,01$; IC à $95 \%: 1,09$ à 3,72) ou de la marijuana $(\mathrm{RC}=2,63$; IC à $95 \%: 1,73$ à 4,01).

Conclusion : Les cigarettes roulées continuent d'être une solution de rechange abordable pour les jeunes fumeurs. Les programmes de prévention ciblant les consommateurs de cigarettes roulées en milieu scolaire pourraient contribuer à réduire la consommation de ce type de cigarettes.

Mots-clés : tabac à rouler, adolescent, tabagisme, consommation d'alcool, consommation de marijuana

\section{Introduction}

Malgré une baisse substantielle des ventes de cigarettes industrielles dans les dernières années, les ventes de produits du tabac plus abordables comme le tabac à rouler ont connu une hausse dans l'ensemble de la population ${ }^{1}$. D'après certaines études, la plupart des fumeurs fument des cigarettes roulées car elles sont moins chères ${ }^{2-4}$. Cette substitution constitue un obstacle sérieux aux politiques actuelles de santé publique qui tentent de décourager le tabagisme par la taxation, d'autant plus que le tabac utilisé pour rouler des cigarettes est généralement plus économique que les cigarettes industrielles ${ }^{5}$. Il est important de connaître la prévalence de la consommation de tabac à rouler chez les jeunes, car il s'agit de la population de fumeurs qui se préoccupe le plus du coût ${ }^{6}$.

Les cigarettes roulées sont roulées à la main avec du tabac à rouler et du papier à cigarette, et sont ensuite munies ou non d'un filtre $^{7}$. Les données probantes indiquent que les cigarettes roulées sont au moins aussi dangereuses que les cigarettes industrielles ${ }^{8-9}$ et qu'elles contribuent au développement de nombreux cancers ${ }^{10-12}$. $\mathrm{Au}$ Canada, les données sur cette consommation sont peu nombreuses, surtout concernant les jeunes. Dans l'Enquête de surveillance de l'usage du tabac au Canada de 2002, $17 \%$ des fumeurs adultes de plus de 15 ans ont déclaré fumer des cigarettes roulées ${ }^{13}$, et les données de l'Enquête sur le tabagisme chez les jeunes (ETJ) de 2008-2009 indiquent que la consommation de cigarettes roulées est actuellement plus élevée chez les jeunes $(24 \%)^{14}$.

Comme la plupart des études ont été axées sur la consommation de tabac à rouler chez les fumeurs adultes, on dispose de peu de données sur les facteurs liés à cette consommation chez les jeunes. L'une des constatations les plus fréquentes est que les fumeurs adultes fumant des cigarettes roulées ont plus souvent un plus faible revenu $^{7,13,15,16}$ et sont plus fréquemment de gros fumeurs ${ }^{4,13,15}$. Les consommateurs de tabac à rouler sont aussi moins nombreux à envisager d'arrêter de fumer ou à avoir récemment tenté de renoncer au tabac que les utilisateurs de cigarettes industrielles ${ }^{4,13,16}$. Les autres facteurs sociodémographiques associés à la consommation de cigarettes roulées chez les fumeurs adultes sont un plus jeune âge, être de sexe masculin et avoir beaucoup d'amis fumeurs ${ }^{4,7,16,17}$.

Comme ce qui s'observe chez les fumeurs adultes, la population de jeunes consommateurs de cigarettes roulées est plus souvent plus jeune et masculine ${ }^{14,17}$ et son revenu disponible est souvent moins

1. École de santé publique et des systèmes de santé, Université de Waterloo, Waterloo (Ontario), Canada 2. Centre pour l'avancement de la santé des populations Propel, Université de Waterloo, Waterloo (Ontario), Canada

Correspondance : Scott T. Leatherdale, School of Public Health and Health Systems, University of Waterloo, 200 University Avenue, Waterloo (Ontario) N2L 3G1; tél. : 519-888-4567, poste 37812; téléc. : 519-746-2510; courriel : sleather@uwaterloo.ca 
élevé $^{14}$. Comparativement aux fumeurs adultes de tabac à rouler, qui préfèrent un produit du tabac aux autres, les jeunes et les jeunes adultes sont plus susceptibles de fumer aussi bien des cigarettes que du tabac à rouler ${ }^{7,16}$. Les jeunes fumant des cigarettes roulées sont aussi plus souvent consommateurs réguliers de marijuana ${ }^{14}$. Si l'on en croit les données internationales, la consommation de tabac à rouler serait non seulement à la hausse chez les jeunes $^{17}$, mais elle serait également plus importante chez les jeunes que dans n'importe quel autre groupe d'âge ${ }^{7}$. C'est pourquoi il est nécessaire d'évaluer les changements survenus dans la prévalence de la consommation de tabac à rouler chez les jeunes Canadiens : cela contribuera à inspirer les futures politiques de lutte antitabac.

L'objectif de cette étude est de caractériser, à l'aide des données représentatives nationales les plus récentes recueillies auprès de jeunes Canadiens fumeurs de la $9^{\mathrm{e}}$ à la $12^{\mathrm{e}}$ année, les variations de prévalence de la consommation de tabac à rouler des jeunes Canadiens entre 2008 et 2010 et d'analyser les caractéristiques sociodémographiques qui leur sont associées.

\section{Méthodologie}

Cette étude s'appuie sur des données représentatives recueillies dans le cadre des cycles 2008-2009 et 2010-2011 de l'ETJ. Des renseignements détaillés sur le plan d'échantillonnage, la méthodologie employée et les taux de participation au cycle 2008-2009 de l'ETJ ont été publiés ailleurs $^{18}$ et sont disponibles sur Internet (www.yss.uwaterloo.ca). Le cycle 20102011 de l'ETJ a permis de recueillir des données auprès de 31396 élèves de la $9^{\mathrm{e}}$ à la $12^{\mathrm{e}}$ année au Canada. La population ciblée se composait des jeunes résidents canadiens de la $9^{\mathrm{e}}$ à la $12^{\mathrm{e}}$ année qui fréquentaient une école secondaire publique, privée ou catholique de l'une des neuf provinces canadiennes participant à l'enquête. Comme le Nouveau-Brunswick a participé à tous les cycles de l'ETJ mais non au cycle 2010-2011, les jeunes de cette province ont été exclus des populations cibles en 2010, et leurs réponses ont été éliminées de l'ensemble de données de 2008 avant l'analyse. Les jeunes du Yukon, du Nunavut et des Territoires du NordOuest, les jeunes vivant en institution ou dans une réserve des Premières Nations et ceux qui fréquentaient une école spécialisée ou une école située dans une base militaire ont aussi été exclus de l'étude. L'ETJ a été administrée aux élèves durant la classe et les participants n'ont reçu aucune rémunération. Le plan d'enquête et la pondération des échantillons ont permis d'obtenir les estimations dans la population présentées dans cet article.

Une combinaison de protocoles d'information active et d'autorisation passive et de protocoles d'autorisation active a servi à recruter les élèves du cycle 2010-2011 de l'ETJ. Les élèves pouvaient aussi refuser de participer à l'enquête le jour de la collecte des données. Le bureau d'éthique de la recherche de l'Université de Waterloo, les commissions scolaires concernées et les comités d'éthique en santé publique ont approuvé toutes les procédures, y compris le consentement passif.

En conformité avec les définitions du tabagisme de Santé Canada élaborées dans le cadre de l'ETJ $^{18}$, nous avons défini comme fumeurs quotidiens les jeunes ayant fumé au moins 100 cigarettes au cours de leur vie et au moins une cigarette entière quotidiennement dans les 30 jours précédant l'enquête, comme fumeurs occasionnels les jeunes ayant fumé au moins 100 cigarettes au cours de leur vie et au moins une cigarette entière au cours des 30 jours précédant l'enquête mais n'ayant pas fumé tous les jours. Nous avons mesuré la consommation de tabac à rouler parmi les fumeurs à l'aide d'une question subdivisée en sous-questions sur l'utilisation de produits du tabac autres que les cigarettes industrielles : "Au cours des 30 derniers jours, as-tu utilisé l'un des produits suivants? (Coche toutes les réponses qui s'appliquent.) " Pour cette analyse, les données sur la consommation de tabac à rouler ont été considérées comme manquantes lorsque les répondants n'avaient rien coché.

L'ETJ a aussi permis de recueillir des données personnelles et des renseignements sur l'argent disponible chaque semaine ainsi que sur la consommation d'alcool et de marijuana. L'une des ques- tions mesurait le revenu disponible du répondant : «Combien d'argent as-tu par semaine pour tes dépenses personnelles ou tes économies? (Pense à inclure l'argent des allocations, des emplois comme la livraison de journaux, etc.) " Conformément aux définitions antérieures ${ }^{19,20}$, les non-buveurs ont été définis comme des jeunes n'ayant déclaré aucune consommation d'alcool au cours de la dernière année, les buveurs occasionnels, comme des jeunes ayant déclaré une consommation mensuelle et les buveurs réguliers, comme des jeunes ayant déclaré une consommation hebdomadaire. De même, les non-consommateurs de marijuana sont ceux n'ayant déclaré aucune consommation de marijuana au cours de la dernière année, les consommateurs occasionnels ceux ayant déclaré une consommation mensuelle et les consommateurs réguliers, une consommation hebdomadaire.

Nous avons appliqué des poids de sondage dans les statistiques descriptives pour tenir compte des différences de taux de réponse selon les régions et les groupes. Comme cela a été décrit précédemment $^{18}$, la construction des poids de sondage s'est effectuée en deux temps. Dans un premier temps, un coefficient de pondération $\left(\mathrm{W}_{1 \mathrm{j}}\right)$ a été créé pour tenir compte du choix des écoles dans les strates relatives à la région sanitaire et au type d'établissement (école élémentaire ou école secondaire selon l'effectif par année scolaire). Un second coefficient de pondération $\left(W_{2 j g}\right)$ a été calculé pour tenir compte des élèves qui n’ont pas répondu. Les facteurs de pondération ont ensuite été calibrés en fonction de la distribution provinciale des élèves selon le sexe et l'année scolaire, afin que les totaux pondérés selon le sexe, l'année scolaire et la province soient égaux à l'effectif réel de ces groupes.

Nous avons réalisé les analyses descriptives des caractéristiques de l'échantillon en fonction de l'année de cueillette de données. Nous avons ensuite eu recours à un modèle de régression logistique utilisant les données de 2010, pour étudier les facteurs associés à la consommation de cigarettes roulées. Nous avons utilisé le système d'analyse SAS version 9.2 pour toutes nos analyses ${ }^{21}$. 


\section{Résultats}

\section{Statistiques descriptives de la consommation de tabac à rouler}

Sur l'ensemble des jeunes de la $9^{\mathrm{e}}$ à la $12^{\mathrm{e}}$ année, 30,5 \% de ceux qui fumaient des cigarettes industrielles ont déclaré fumer aussi des cigarettes roulées, 15,3\% des jeunes ayant fumé auparavant des cigarettes industrielles ont déclaré fumer actuellement des cigarettes roulées et 1,0\% des jeunes n'ayant jamais fumé de cigarettes industrielles ont déclaré fumer des cigarettes roulées. Le tableau 1 présente les résultats pondérés des statistiques descriptives de la consommation de tabac à rouler en 2010 chez les jeunes de la $9^{\mathrm{e}}$ à la $12^{\mathrm{e}}$ année.

La figure 1 présente la proportion d'élèves qui ont déclaré fumer des cigarettes roulées, selon l'année scolaire et le cycle de l'ETJ. Autant dans le cycle de 2008 que dans celui de 2010, la prévalence de la consommation de cigarettes roulées était plus élevée chez les élèves de la $12^{\mathrm{e}}$ année que chez les élèves de la $9^{\mathrm{e}}$ année. Dans l'ensemble, la prévalence de l'utilisation de tabac à rouler a diminué d'environ $5 \%$ entre 2008 et 2010, bien que cette variation ne soit pas significative $\left(\chi^{2}=1,4 ; p>0,05\right.$; $d f=1)$.

En 2008 comme en 2010, le recours aux cigarettes roulées était invariablement plus fréquent dans la région du Canada atlantique et en Colombie-Britannique et était systématiquement moins fréquent au Québec et en Ontario. Entre 2008 et 2010, la consommation de cigarettes roulées a baissé en Colombie-Britannique et a augmenté dans la région du Canada atlantique et au Québec, alors qu'elle est demeurée relativement stable en Ontario et dans les Prairies (figure 2).

\section{Résultats du modèle de régression}

logistique concernant la consommation de cigarettes roulées

Les résultats pondérés du modèle de régression visant à analyser la consommation de cigarettes roulées chez les jeunes fumeurs sont présentés dans le tableau 2. La différence entre les sexes pour ce qui est des cotes de consommation et de nonconsommation de cigarettes roulées n'était

TABLEAU 1

Résultats pondérés des statistiques descriptives de la consommation de tabac à rouler au sein de l'échantillon de fumeurs $\left(9^{\mathrm{e}}\right.$ à $12^{\mathrm{e}}$ année) du cycle 2010-2011 de l'Enquête sur le tabagisme chez les jeunes au Canada

\begin{tabular}{cc} 
Paramètres & $\begin{array}{c}\text { Consommation de tabac à } \\
\text { rouler }(n=41900)^{\mathrm{a}}\end{array}$ \\
\cline { 2 - 3 } & Pourcentage d'élèves
\end{tabular}$\quad$ Khi carré

\section{Sexe}

Fille

Garçon

38,6

61,4

Année scolaire

$9^{\mathrm{e}}$

$10^{\mathrm{e}}$

18,0

29,0

$11^{\mathrm{e}}$

27,6

$12^{\mathrm{e}}$

25,5

Région

Canada atlantique ${ }^{\mathrm{b}}$

Québec

11,2

Ontario

$18,4^{\mathrm{E}}$

Prairies $^{c}$

Colombie-Britannique

Origine ethnique

Blanc

Noir, Latino-Américain, autre

65,6

18,3

Asiatique

3,7

Autochtone

12,4

Catégorie de fumeur

Fumeur quotidien $^{\mathrm{d}}$

Fumeur occasionnel ${ }^{\mathrm{e}}$

62,4

37,6

Nombre moyen de cigarettes fumées quotidiennement dans les 30 derniers jours (n)

$\begin{array}{lll}5 \text { ou moins } & 37,1 & \chi^{2}=155,6 ; p<0,001 ; d f=2 \\ 6 \text { à } 10 & 28,0 & \\ 11 \text { ou plus } & 34,9 & \end{array}$

Tentative(s) d'abandonner la cigarette (n)

0

1

2 ou plus

37,3

$\chi^{2}=19,5 ; p<0,001 ; d f=2$

Raisons pour lesquelles le répondant fume la marque de cigarettes déclarée $\mathrm{f}^{\mathrm{f}}$
Mes amis fument la même marque.

La marque coûte moins cher que les autres.

J'aime l'image que projette la marque.

J'aime le goût.

Utilisation de feuilles d'enveloppe

Oui

Non $\chi^{2}=9,4 ; p<0,01 ; d f=1$

$\chi^{2}=84,2 ; p<0,001 ; d f=3$

$\chi^{2}=84,9 ; p<0,001 ; d f=4$

23,7

23,8

23,0

\section{6}

$\chi^{2}=33,8 ; p<0,001 ; d f=3$

Utilisation de feuilles d'enveloppe dans les 30 derniers jours

Oui $\quad 30,7$

Non
69,3

$\begin{array}{rc}14,6 & \chi^{2}=6,6 ; p<0,05 ; d f=1 \\ 23,1 & \chi^{2}=0,2 ; p>0,05 ; d f=1 \\ 6,3 & \chi^{2}=14,7 ; p<0,001 ; d f=1 \\ 67,2 & \chi^{2}=8,0 ; p<0,01 ; d f=1\end{array}$

46,7

$\chi^{2}=157,2 ; p<0,001 ; d f=1$

53,3

30,7

$\chi^{2}=181,6 ; p<0,001 ; d f=1$

Suite page suivante 
TABLEAU 1 (Suite)

Résultats pondérés des statistiques descriptives de la consommation de tabac à rouler au sein de l'échantillon de fumeurs ( $9^{\mathrm{e}}$ à $12^{\mathrm{e}}$ année) du cycle 2010-2011 de l'Enquête sur le tabagisme chez les jeunes au Canada

\begin{tabular}{|c|c|c|}
\hline \multirow[t]{2}{*}{ Paramètres } & $\begin{array}{l}\text { Consommation de tabac à } \\
\text { rouler }(\mathrm{n}=41900)^{\mathrm{a}}\end{array}$ & \multirow[t]{2}{*}{ Khi carré } \\
\hline & Pourcentage d'élèves & \\
\hline \multicolumn{3}{|c|}{ Nombre d'amis proches qui fument la cigarette (n) } \\
\hline 0 & $3,0^{\mathrm{E}}$ & $\chi^{2}=133,5 ; p<0,001 ; d f=5$ \\
\hline 1 & $2,2^{\mathrm{E}}$ & \\
\hline 2 & 6,0 & \\
\hline 3 & 6,4 & \\
\hline 4 & 4,3 & \\
\hline 5 et plus & 78,6 & \\
\hline \multicolumn{3}{|c|}{ Argent dont disposent chaque semaine les répondants pour dépenser ou pour économiser (\$) } \\
\hline 0 & 11,2 & $\chi^{2}=4,0 ; p>0,05 ; d f=4$ \\
\hline 1 à 20 & 26,2 & \\
\hline 21 à 100 & 29,0 & \\
\hline Plus de 100 & 24,4 & \\
\hline Ne sait pas & 9,1 & \\
\hline \multicolumn{3}{|c|}{ Consommation d'alcool dans les 12 derniers mois } \\
\hline Aucune $^{g}$ & 4,1 & $\chi^{2}=31,1 ; p<0,001 ; d f=2$ \\
\hline Occasionnelle $^{\mathrm{h}}$ & 44,8 & \\
\hline Régulière $^{\mathrm{i}}$ & 51,1 & \\
\hline \multicolumn{3}{|c|}{ Consommation de marijuana dans les 12 derniers mois } \\
\hline Aucune ${ }^{g}$ & 5,7 & $\chi^{2}=91,7 ; p<0,001 ; d f=2$ \\
\hline Occasionnelle $^{\mathrm{h}}$ & 22,1 & \\
\hline Régulière $^{\mathrm{i}}$ & 72,2 & \\
\hline
\end{tabular}

a Estimation pondérée de l'échantillon (voir partie méthodologie).

b La région du Canada atlantique comprend Terre-Neuve-et-Labrador, l'île-du-Prince-Édouard et la Nouvelle-Écosse (le Nouveau-Brunswick ne faisait pas partie de l'échantillon de l'ETJ 2010-2011).

c La région des Prairies comprend le Manitoba, la Saskatchewan et l'Alberta.

d Les fumeurs quotidiens ont fumé au moins 100 cigarettes au cours de leur vie et au moins une cigarette entière par jour pendant les 30 jours précédant l'enquête.

e Les fumeurs occasionnels ont fumé au moins 100 cigarettes au cours de leur vie et au moins une cigarette entière dans les 30 jours précédant l'enquête, mais n'ont pas fumé tous les jours.

${ }^{f}$ Les répondants pouvaient choisir plus d'une réponse.

${ }^{g}$ Les non-consommateurs ont déclaré aucune consommation au cours de l'année précédente.

${ }^{\mathrm{h}}$ Les consommateurs occasionnels ont déclaré une consommation mensuelle.

i Les consommateurs réguliers ont déclaré une consommation hebdomadaire.

E Variabilité modérée de l'échantillonnage, à interpréter avec prudence.

pas significative $(\mathrm{RC}=1,09$, IC à $95 \%$ : $0,88$ à 1,36$)$. Les élèves de la $10^{\mathrm{e}}$, de la $11^{\mathrm{e}}$ et de la $12^{\mathrm{e}}$ année étaient significativement moins nombreux à fumer des cigarettes roulées que les élèves de $9^{\mathrm{e}}$ année $(\mathrm{RC}=0,67$, IC à $95 \%: 0,48$ à 0,$95 ; \mathrm{RC}=0,53$, IC à $95 \%: 0,38$ à 0,73 et RC $=0,29$, IC à $95 \%$ : 0,20 à 0,42 , respectivement). Par ailleurs, comparativement aux jeunes qui ne fu- maient pas de cigarettes roulées, les fumeurs de cigarettes roulées étaient moins nombreux à fumer seulement occasionnellement $(\mathrm{RC}=0,47$, IC à $95 \%: 0,37$ à 0,58$)$, à avoir essayé d'arrêter de fumer une fois $(\mathrm{RC}=0,62$, IC à $95 \%: 0,47$ à 0,83$)$ ou deux fois ou plus $(\mathrm{RC}=0,61$, IC à $95 \%$ : 0,48 à 0,78 ) et à avoir généralement un revenu disponible variant entre 21 \$ et 100 \$
$(\mathrm{RC}=0,61, \mathrm{IC}$ à $95 \%: 0,42$ à 0,87$)$ ou de plus de $100 \$$ par semaine $(\mathrm{RC}=0,49$, IC à $95 \%: 0,34$ à 0,71). En revanche, ces fumeurs étaient plus nombreux à se décrire comme étant noirs, latino-américains ou autre $(\mathrm{RC}=1,54$, IC à $95 \%: 1,13$ à 2,11), à fumer des cigarettes roulées parce qu'ils aimaient l'image de la marque $(\mathrm{RC}=1,82$, IC à $95 \%: 1,11$ à 2,99), à avoir déjà essayé d'utiliser des feuilles d'enveloppe* (RC = 2,61 , IC à $95 \%: 2,05$ à 3,31), à boire occasionnellement (RC $=2,69$, IC à $95 \%$ : $1,46$ à 4,96$)$ ou régulièrement $(\mathrm{RC}=2,01$, IC à $95 \%: 1,09$ à 3,72) et à consommer occasionnellement (RC = 3,09, IC à $95 \%$ : 1,97 à 4,83 ) ou régulièrement $(\mathrm{RC}=2,63$, IC à $95 \%$ : 1,73 à 4,01) de la marijuana.

\section{Analyse}

Nos données indiquent que la consommation de tabac à rouler, bien qu'elle ne soit pas aussi élevée que celle de cigarettes industrielles, n'est pas négligeable au Canada. En effet, en 2010, presque le tiers des jeunes fumeurs (soit plus de 40000 jeunes Canadiens de la $9^{\mathrm{e}}$ à la $12^{\mathrm{e}}$ année) consommaient ce type de produit du tabac. Une comparaison des données de 2008 et de 2010 révèle que la prévalence de la consommation de cigarettes roulées chez les jeunes Canadiens est restée relativement stable, ce qui reflète peutêtre la stabilité des taux de consommation de cigarettes industrielles ${ }^{22}$. Il est néanmoins clair que la consommation de cigarettes roulées chez les jeunes demeure un problème de santé grave et devrait continuer à être l'objet de programmes de prévention du tabagisme et de renoncement au tabac.

Nos données, qui concordent avec celles des publications antérieures, indiquent que les jeunes ayant un revenu disponible élevé sont moins nombreux à fumer des cigarettes roulées. Alors que la taxation demeure une méthode efficace pour réduire la prévalence du tabagisme chez les jeunes ${ }^{23}$, les cigarettes roulées demeurent généralement plus économiques que les cigarettes industrielles ${ }^{24}$. Dans huit des dix provinces canadiennes, le tabac à rouler est en effet deux fois moins taxé

\footnotetext{
* Papier à cigarette composé de tabac.
} 
FIGURE 1

Proportion de jeunes consommateurs de tabac à rouler par année scolaire selon les cycles 2008-2009 et 2010-2011 de l'Enquête sur le tabagisme chez les jeunes au Canada

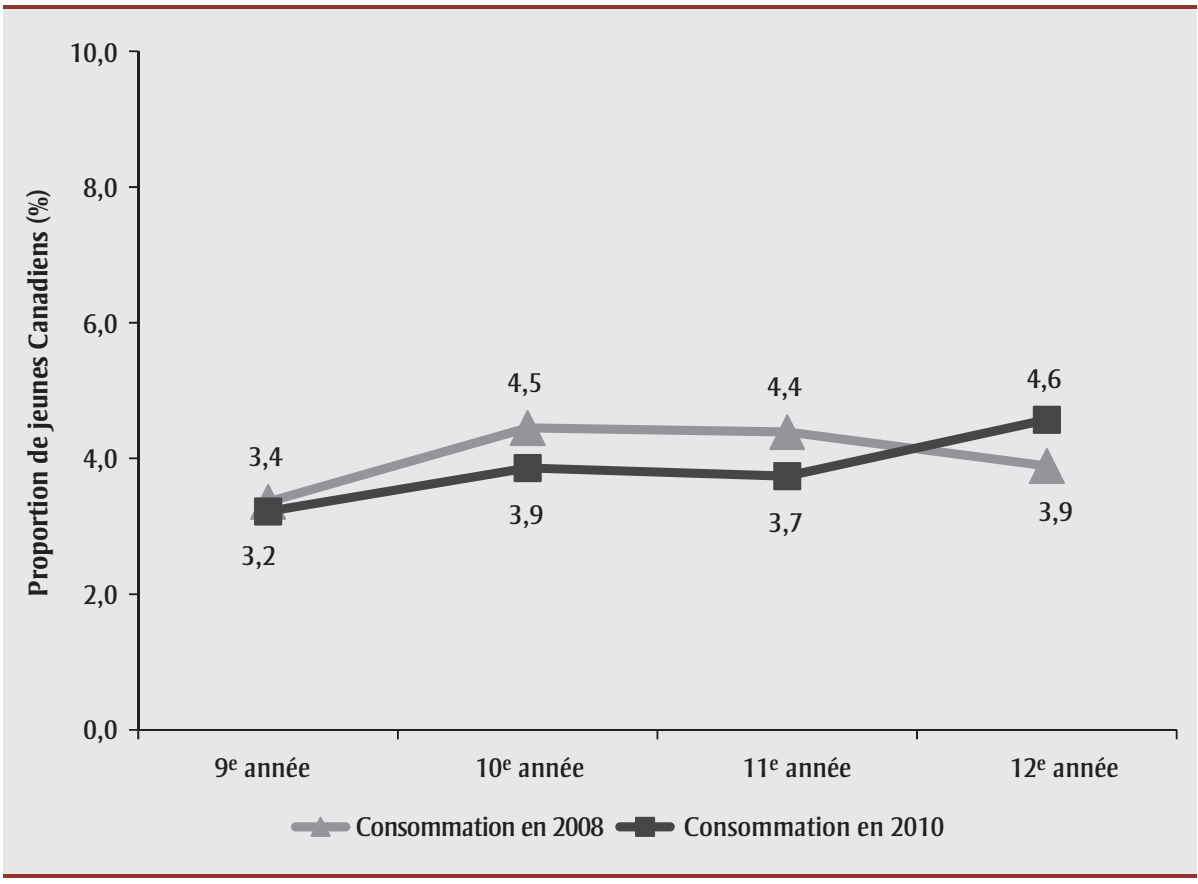

FIGURE 2

Proportion de jeunes consommateurs de tabac à rouler par région selon les cycles 2008-2009 et 2010-2011 de l'Enquête sur le tabagisme chez les jeunes au Canada

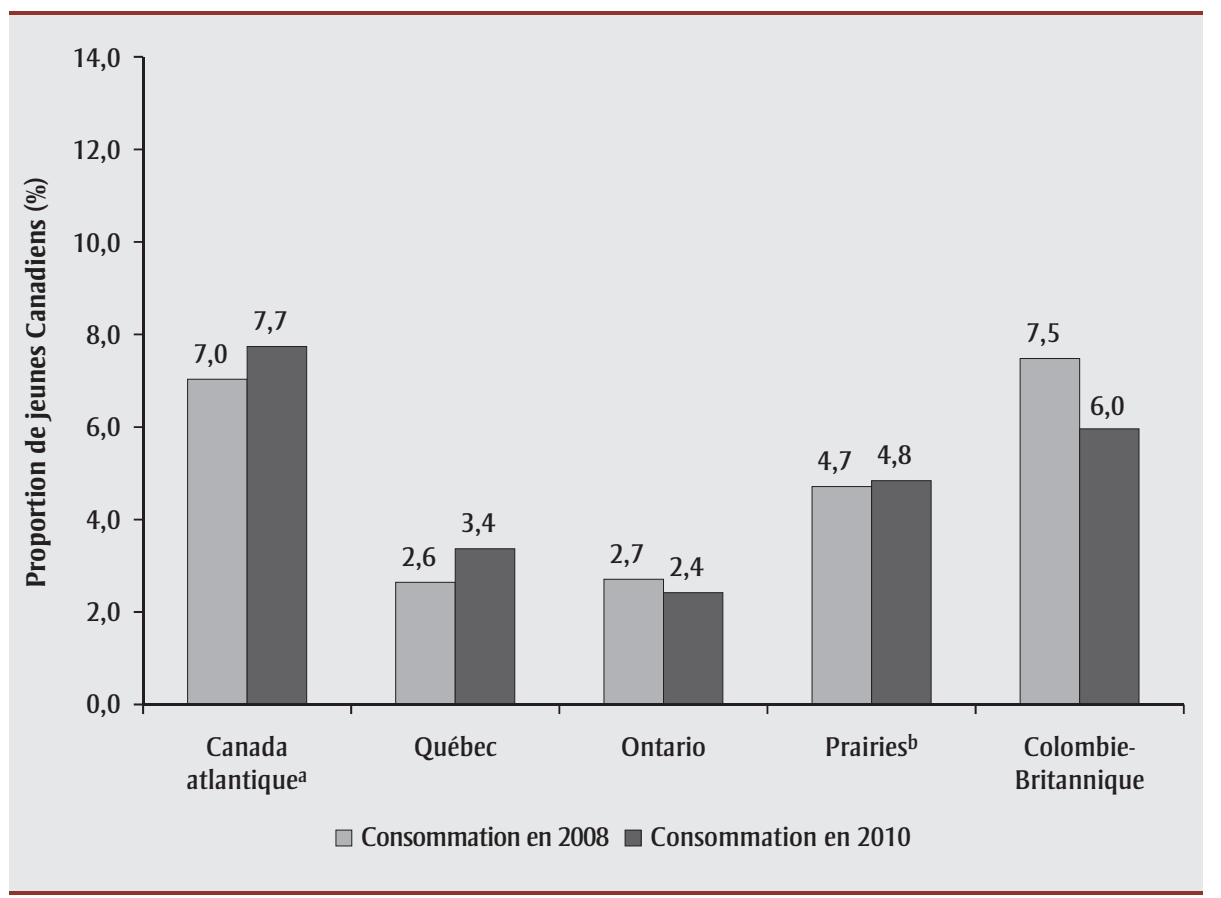

${ }^{a}$ La région du Canada atlantique comprend Terre-Neuve-et-Labrador, l'île-du-Prince-Édouard et la Nouvelle-Écosse (le Nouveau-Brunswick ne faisait pas partie de l'échantillon de l'ETJ 2010-2011).

${ }^{\text {b }}$ La région des Prairies comprend le Manitoba, la Saskatchewan et l'Alberta. que les cigarettes industrielles ${ }^{5}$, ce qui rend les cigarettes roulées plus abordables. Nous recommandons donc que le tabac à rouler soit davantage taxé, afin de réduire la différence de prix entre les cigarettes roulées et les cigarettes industrielles ${ }^{\dagger}$.

En accord avec les travaux de recherche antérieurs, il ressort de nos données que les jeunes qui fument des cigarettes roulées consomment plus fréquemment de la marijuana, de l'alcool et des feuilles d'enveloppe ${ }^{25}$. D'autres études indiquent que les jeunes qui mélangent tabac et marijuana dans une feuille d'enveloppe roulée sont davantage susceptibles non seulement de devenir dépendants de ces deux substances, mais également de consommer d'autres drogues illicites comme la cocaïne et des drogues hallucinogènes ${ }^{26}$. Les programmes de prévention en milieu scolaire visant la consommation de plusieurs substances demeurent donc pertinents et nécessaires ${ }^{27}$. En outre, comme les jeunes moins âgés sont plus nombreux à fumer des cigarettes roulées que les jeunes plus âgés, les programmes axés sur les jeunes de $9^{\mathrm{e}}$ et de $10^{\mathrm{e}}$ année pourraient être plus efficaces pour prévenir la consommation des cigarettes roulées. Ces programmes devraient cibler spécifiquement la consommation de cigarettes roulées, car les recherches indiquent que les programmes antitabac ciblés sont plus efficaces que les programmes contre la consommation abusive de drogues en général $^{28}$. Il faudrait obtenir des données supplémentaires sur l'efficacité de ces approches ciblées.

\section{Points forts et limites}

Cette étude a plusieurs points forts. L'ETJ est une enquête menée tous les deux ans représentative de la population canadienne, qui fournit des données sur les différences de consommation de tabac entre les provinces au Canada et qui permet aux chercheurs de surveiller les variations de la prévalence au fil du temps. Cependant, étant donné la nature transversale des données, l'étude présente

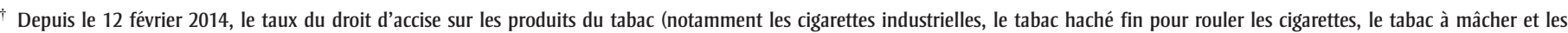
cigares) a auǵmenté pour tenir compte des variations de l'inflation depuis 2002. Cette hausse de taxe est considérée comme positive, mais il existe encore une différence considérable entre le prix des cigarettes roulées et celui des cigarettes industrielles, surtout entre les provinces.
} 
TABLEAU 2

Modèle de régression logistique visant à examiner les facteurs associés à la consommation de tabac à rouler chez les jeunes fumeurs ( $9^{\mathrm{e}}$ à $12^{\mathrm{e}}$ année) du cycle 2010-2011 de l'Enquête sur le tabagisme chez les jeunes au Canada

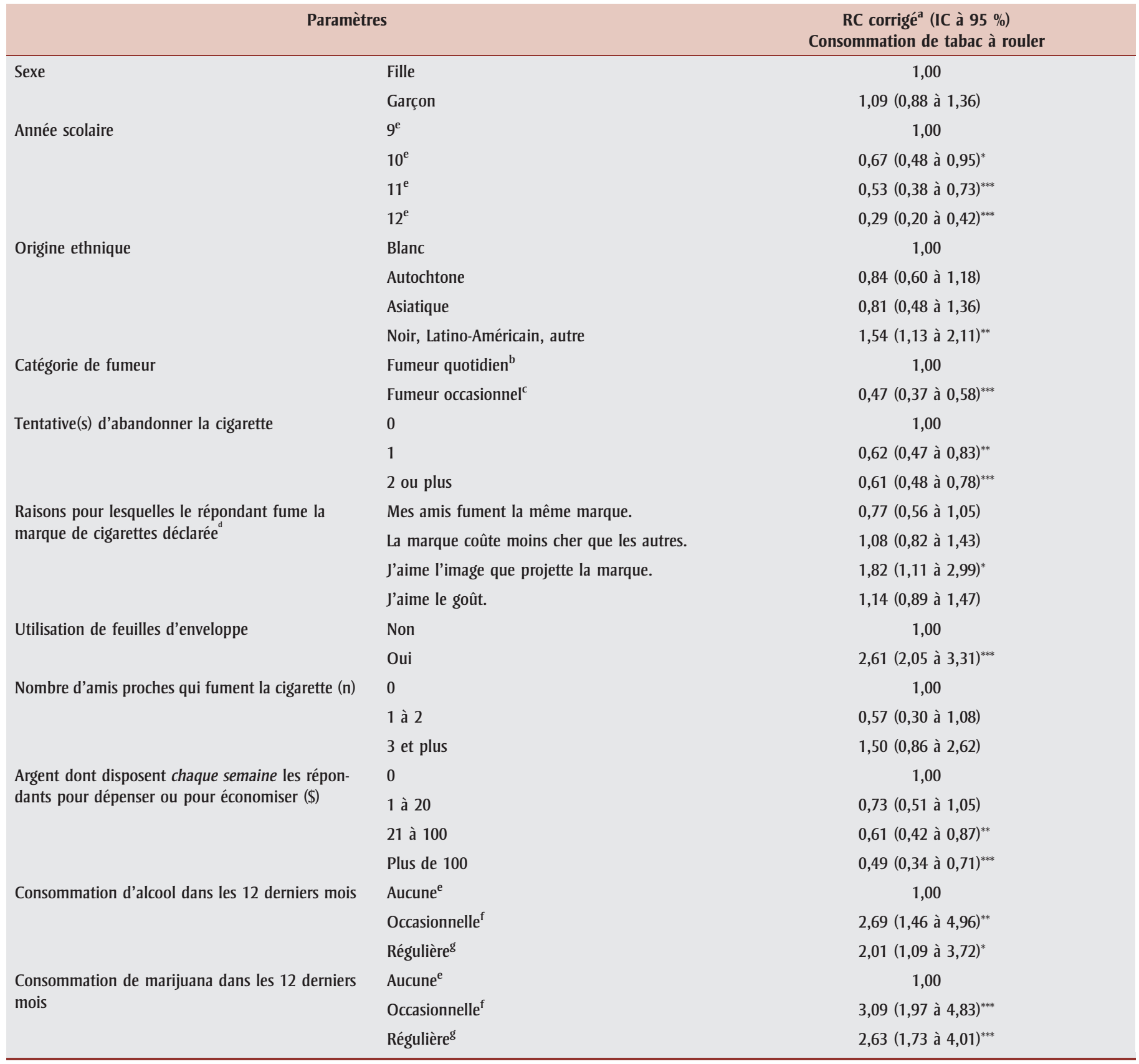

Abréviations : IC, intervalle de confiance; RC, rapport de cotes.

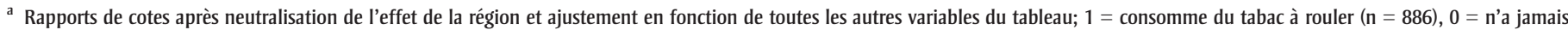
consommé de tabac à rouler $(n=1466)$.

b Les fumeurs quotidiens ont fumé au moins 100 cigarettes au cours de leur vie et au moins une cigarette entière par jour pendant les 30 jours précédant l'enquête.

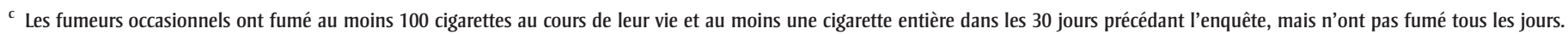

d Le groupe de référence comprend ceux qui n'ont pas répondu et ceux qui ont répondu " non».

e Les non-consommateurs ont déclaré aucune consommation au cours de l'année précédente.

${ }^{\mathrm{f}}$ Les consommateurs occasionnels ont déclaré une consommation mensuelle.

8 Les consommateurs réguliers ont déclaré une consommation hebdomadaire.

${ }^{*} p<0,05$.

** $p<0,01$.

*** $p<0,001$. 
aussi certaines limites. Il est impossible de faire des inférences causales pour ce qui est des variables et de la consommation de tabac à rouler. Par ailleurs, des données longitudinales seraient nécessaires pour déterminer si la consommation de cigarettes roulées précède la consommation de marijuana ou d'alcool ou y conduit. De plus, comme les données utilisées sont fondées sur des comportements autodéclarés liés au tabagisme, la validité des réponses ne peut être garantie, et il pourrait exister un certain biais dans les estimations à cause des élèves qui n'ont pas répondu. Toutefois, il a déjà été démontré que les mesures de la consommation autodéclarée de tabac sont fiables et valides ${ }^{29,30}$, et on a assuré aux élèves que leurs réponses resteraient confidentielles.

\section{Conclusion}

Nos données indiquent que l'utilisation du tabac à rouler n'est pas un problème négligeable chez les jeunes Canadiens. Les jeunes fumeurs de cigarettes roulées disposent plus souvent d'un revenu disponible inférieur, mais ils sont plus nombreux à consommer aussi des feuilles d'enveloppe, de l'alcool et de la marijuana. On peut en faire davantage pour décourager l'utilisation du tabac à rouler, notamment en augmentant les taxes sur ce produit afin de réduire la différence de prix entre les cigarettes industrielles et les cigarettes roulées. Par ailleurs, des programmes de prévention ciblés sur le milieu scolaire visant la consommation de cigarettes roulées pourraient contribuer à diminuer davantage la consommation de ce type de produit.

\section{Remerciements}

L'ETJ est le fruit d'un projet pancanadien de renforcement des capacités financé dans le cadre d'un accord de contribution entre Santé Canada et le Centre pour l'avancement de la santé des populations Propel de l'Université de Waterloo. Ce consortium pancanadien réunit des chercheurs de toutes les provinces œuvrant dans le domaine de la lutte contre le tabagisme et offre des possibilités de formation à des étudiants universitaires de tous les cycles, encourageant leur partici- pation et leur spécialisation dans le domaine de la recherche sur la lutte antitabac. La rédaction de cet article a été rendue possible grâce à une contribution financière de l'Unité de recherche sur le tabac de l'Ontario.

\section{Références}

1. Connolly GN, Alpert HR. Trends in the use of cigarettes and other tobacco products, 20002007. JAMA. 2008;299(22):2629-2630.

2. Nosa V, Glover M, Min S et collab. The use of the 'rollie' in New Zealand: preference for loose tobacco among an ethnically diverse low socioeconomic urban population. N Z Med J. 2011;124(1338):25-33.

3. Wilson N, Young D, Weerasekera D, Edwards R, Thomson G, Glover M. The importance of tobacco prices to roll-yourown (RYO) smokers (national survey data): higher tax needed on RYO. N Z Med J. 2009;122(1305):92-96.

4. Young D, Wilson N, Borland R, Edwards R, Weerasekera D. Prevalence, correlates of, and reasons for using roll-your-own tobacco in a high RYO use country: findings from the ITC New Zealand Survey. Nicotine Tob Res. 2010;12(11):1089-1098.

5. Ontario Tobacco Research Unit. Tobacco taxes : monitoring update. Monitoring and Evaluation Series (vol. 16, $\mathrm{n}^{\circ} 1$ ). Toronto (Ont.) : Ontario Tobacco Research Unit; 2010.

6. Chaloupka FJ, Straif K, Leon ME. Effectiveness of tax and price policies in tobacco control. Tob Control. 2011;20(3): 235-238.

7. Li J, Grigg M, Weerasekera D et collab. Characteristics and quitting success of rollyour-own versus tailor-made cigarette smokers. N Z Med J. 2010;123(1308):64-73.

8. Laugesen $M$, Epton $M$, Frampton $C M$, Glover M, Lea RA. Hand-rolled cigarette smoking patterns compared with factorymade cigarette smoking in New Zealand men. BMC Public Health. 2009;9:194-199. doi : 10.1186/1471-2458-9-194

9. Shahab L, West R, McNeill A. A comparison of exposure to carcinogens among rollyour-own and factory-made cigarette smo- kers. Addict Biol. 2009;14(3):315-320. doi : 10.1111/j.1369-1600.2009.00157.x

10. Tuyns AJ, Esteve J. Pipe, commercial and hand-rolled cigarette smoking in oesophageal cancer. Int J Epidemiol. 1983;12(1): 110-113.

11. Engeland A, Haldorsen T, Andersen A, Tretli S. The impact of smoking habits on lung cancer risk: 28 years' observation of 26,000 Norwegian men and women. Cancer Causes Control. 1996;7:366-376.

12. De Stefani E, Oreggia F, Rivero S, Fierro L. Hand-rolled cigarette smoking and risk of cancer of the mouth, pharynx, and larynx. Cancer. 1992;70(3):679-682.

13. Leatherdale ST, Kaiserman M, Ahmed R. The roll-your-own cigarette market in Canada: a cross-sectional exploratory study. Tob Induc Dis. 2009;5(5):1-6. doi : 10.1186/1617-9625-5-5

14. Leatherdale ST, Burkhalter R. Roll-yourown tobacco use among Canadian youth : is it a bigger problem than we think? BMC Public Health. 2012;12:557. doi : 10.1186 /1471-2458-12-557

15. Licht AS, Hyland AJ, O'Connor RJ et collab. Socio-economic variation in price minimizing behaviors: findings from the International Tobacco Control (ITC) Four Country Survey. Int J Environ Res Public Health. 2011;8(1):234-252.

16. Young D, Borland R, Hammond D et collab. Prevalence and attributes of roll-your-own smokers in the International Tobacco Control (ITC) Four Country Survey. Tob Control. 2006;15(Suppl 3):iii76-82.

17. Raisamo SU. Trends in roll-your-own tobacco use among adolescents in Finland, 1981-2009. Prev Med. 2011;53(6):431-432.

18. Elton-Marshall T, Leatherdale ST, Manske SR, Wong $\mathrm{K}$, Ahmed $\mathrm{R}$, Burkhalter R. Méthodologie de l'Enquête sur le tabagisme chez les jeunes (ETJ). Maladies chroniques et blessures au Canada. 2011;32:53-61.

19. Leatherdale ST, Burkhalter R. The substance use profile of Canadian youth: exploring the prevalence of alcohol, drug and tobacco use by gender and grade. Addict Behav. 2012;37:318-322. 
20. Leatherdale ST, Rynard V. A cross-sectional examination of modifiable risk factors for chronic disease among a nationally representative sample of youth: are Canadian students graduating high school with a failing grade for health? BMC Public Health. 2013;3:569. doi : 10.1186/10.1186 /1471-2458-13-569

21. SAS Institute Inc. The SAS System for Windows: Version 9.2. Cary (NC): SAS Institute Inc.; 2008.

22. Reid JL, Hammond D, Rynard VL, Burkhalter R. Tobacco use in Canada: patterns and trends, 2014 ed. Waterloo (Ont.): Propel Centre for Population Health Impact, University of Waterloo; 2014.

23. Carpenter C, Cook PJ. Cigarette taxes and youth smoking: new evidence from national, state and local Youth Risk Behavior Surveys. J Health Econ. 2008;27(2):287-299.

24. Mindell JS, Whynes DK. Cigarette consumption in The Netherlands 1970-1995: does tax policy encourage the use of handrolling tobacco? Eur J Public Health. 2000;10(3):214-219.

25. Crawford GE. Flavoured tobacco products with marijuana names. Tob Control. 2007; 16(1):70.

26. Timberlake DS. A comparison of drug use and dependence between blunt smokers and other cannabis users. Subst Use Misuse. 2009;44:401-415.

27. Leatherdale ST, Ahmed R. Alcohol, marijuana, and tobacco use among Canadian youth: do we need more multi-substance prevention programming? J Prim Prev. 2010;31:99-108.

28. Tobler NS, Roona MR, Ochshorn P, Marshall DG, Streke AV, Stackpole KM. School-based adolescent drug prevention programs : 1998 meta-analysis. J Prim Prev. 2000;20(4):275-336.

29. Fendrich M, Mackesy-Amiti ME, Johnson TP, Hubbell A, Wislar JS. Tobacco-reporting validity in an epidemiological drug-use survey. Addict Behav. 2005;30:175-181.

30. Messeri PA, Allen JA, Mowery PD et collab. Do tobacco countermarketing campaigns increase adolescent under-reporting of smoking? Addict Behav. 2007;32:1532-1536. 